\title{
Eicosapentaenoic Acid Blocks Cyclosporine A-Induced Pancreatic Dysfunction but not Immunosuppression
}

\author{
Takashi Majima $^{1 *}$, Kazuki Sasaki ${ }^{2}$, Hiroshi Ohara ${ }^{2}$, Norio Takahashi ${ }^{2}$ \\ ${ }^{1}$ Department of Pharmacy Practice and Pharmaceutical Sciences, Setsunan University, Hirakata, Osaka, Japan. \\ ${ }^{2}$ Department of Clinical Pharmacy, School of Pharmaceutical Sciences, Ohu University, Koriyama, Fukushima, Japan.
}

\begin{tabular}{|c|c|}
\hline ARTICLE INFO & ABSTRACT \\
\hline $\begin{array}{l}\text { Article history: } \\
\text { Received on: } 19 / 03 / 2017 \\
\text { Accepted on: } 12 / 05 / 2017 \\
\text { Available online: 30/06/2017 }\end{array}$ & $\begin{array}{l}\text { Cyclosporine A (CsA) is a widely used immunosuppressant in transplantation medicine. However, it has } \\
\text { oxidative stress-induced side effects, including nephrotoxicity, hypertension, dyslipidemia, and glucose } \\
\text { intolerance; particularly, post-transplant diabetes mellitus, accompanied by alterations of pancreatic islet cells, } \\
\text { has been reported as a major adverse event. Recent research indicates that omega-3 polyunsaturated fatty acids, }\end{array}$ \\
\hline $\begin{array}{l}\text { Key words: } \\
\text { CsA, islet cell vacuolation, } \\
\text { glucose intolerance, EPA, } \\
\text { NFAT. }\end{array}$ & $\begin{array}{l}\text { effects of EPA against CsA-induced alterations of pancreatic islet cells and hyperglycemia have not been } \\
\text { examined in long-term administration of CsA in rats. The aim of this study was to determine whether EPA can } \\
\text { abrogate these negative effects by assessing pancreatic islet cell dysfunction, vacuolation, and hyperglycemia in } \\
\text { rats continuously treated with CsA. These pathologies were significantly improved by concomitant } \\
\text { administration of EPA. Importantly, EPA did not affect the immunosuppressive effects of CsA, as evidenced by } \\
\text { nuclear translocation of nuclear factor of activated T-cells in pancreatic tissue in response to lipopolysaccharide } \\
\text { stimulation. Thus, EPA can be used to prevent CsA-induced oxidative stress and/or inflammation in pancreatic } \\
\text { islets. Our data also suggest that EPA can be used in conjunction with CsA to mitigate its harmful side effects } \\
\text { while preserving its immunosuppressant properties in transplant patients. }\end{array}$ \\
\hline
\end{tabular}

\section{INTRODUCTION}

Cyclosporine A (CsA) is widely used in transplantation medicine as an immunosuppressant. This effect is exerted via inhibition of cytokine production [e.g., interleukin (IL)-2] by helper $\mathrm{T}$ cells. However, long-term use of CsA has adverse effects such as nephrotoxicity, hypertension, dyslipidemia, and glucose intolerance (Heisel et al., 2004; Penfornis and KuryPaulin, 2006; Lim et al., 2012; Piao SG et al., 2012). CsAinduced oxidative stress has been found to play a critical role in pancreatic islet dysfunction, including hyperglycemia, reduced insulin levels and islet mass, and increased apoptotic cell death and macrophage infiltration (Gómez et al., 2004; Lim et al., 2013). Post-transplant diabetes mellitus (PTDM) is a major adverse event in $10-25 \%$ of patients receiving immuno-

\footnotetext{
* Corresponding Author

Takashi Majima, Department of Practical Pharmaceutical Sciences, Setsunan University. Email: takashi.majima @ pharm.setsunan.ac.jp
}

suppressive therapy (Drachenberg et al., 1999; Jiang et al., 1999; Gómez et al., 2004; Lim et al., 2013), which can lead to reduced graft survival and increased risk of infectious and cardiovascular diseases. The pathogenesis of PTDM is thought to involve direct toxicity of CsA to pancreatic beta cells and a consequent reduction in insulin synthesis and secretion (Yang et al., 2002; Gómez et al., 2004). Recent studies have shown that chronic CsA administration impairs glucose tolerance and induces nephrotoxicity, which is characterized by striped tubulointerstitial fibrosis, tubular atrophy, and hyalinosis of afferent arterioles, resulting from oxidative stress and inflammation (Hahn et al., 1986, 1992; Düfer et al., 2001; Yang et al., 2002; Justo et al., 2003; Chung et al., 2005; Song et al., 2009; Lim SW et al., 2012; Piao et al., 2012). The search for drugs that can ameliorate CsA-induced adverse side effects related to glucose intolerance is on-going. Omega-3 polyunsaturated fatty acids (PUFAs), including eicosapentaenoic acid (EPA), have antioxidant properties and anti-inflammatory activity, and thus have beneficial effects on cardiovascular health (Shimojo et al., 2006; Weylandt et al., 2008; Oh et al., 2010). 
We hypothesized that EPA could reduce glucose intolerance and thereby prevent organ injury caused by CsA. The aim of this study was to test this hypothesis using a rat model of chronic CsA treatment. We found that co-administration of EPA suppressed the CsA-induced increases in plasma glucose concentration and pancreatic islet cell alterations.

\section{MATERIAL AND METHODS}

\section{Reagents}

Rabbit polyclonal anti-nuclear factor of activated T-cells 3 (NFATc3) (Santa Cruz Biotechnology, Dallas, TX, USA) and rabbit monoclonal anti- $\beta$-actin (13E5) (Cell Signaling Technology, Danvers, MA, USA) antibodies were used in the experiments. TOPRO-3 and Alexa 488-conjugated rabbit IgG were purchased from Life Technologies (Carlsbad, CA, USA). CsA was obtained from LC Laboratories (Boston, MA, USA), and EPA was from $\mathrm{Nu}$ Chek Prep (Elysian, MN, USA).

\section{Animal groups and treatments}

Wistar/ST rats (6-week-old) were purchased from Nihon SLC (Shizuoka, Japan). Rats were housed in a controlled environment under a 12:12-h light/dark cycle with the temperature maintained at $25{ }^{\circ} \mathrm{C}$, and had free access to standard chow and water. Rats were divided into three groups (3-5 rats per group): control, CsA, and CsA + EPA. Control rats received vehicle treatment (saline followed by olive oil), those in the CsA group were treated daily with $40 \mathrm{mg} / \mathrm{kg} \mathrm{CsA}$ in olive oil via gavage, and those in the CsA + EPA group were treated daily with $15 \mathrm{mg} / \mathrm{kg}$ EPA in saline followed by $40 \mathrm{mg} / \mathrm{kg} \mathrm{CsA}$ in olive oil via gavage. Animals were euthanized at the end of the 2-week treatment period.

\section{Intraperitoneal glucose tolerance test (IPGTT)}

The IPGTT was performed on day 15. Briefly, after 1 day of fasting, rats were injected with $20 \%$ d-glucose solution (2 $\mathrm{g} / \mathrm{kg}$ ), with blood glucose concentration measured immediately before and 15, 30, 60, 90, 120, and 180 min after injection with a glucose analyzer (Glutest Neo Super; Sanwa Kagaku Kenkyusho, Nagoya, Japan). The area under the curve of glucose (AUCg) was calculated by trapezoidal estimation from values obtained in the IPGTT.

\section{Histological analysis}

After IPGTT, rats were anesthetized by low-dose diethyl ether inhalation, followed by intraperitoneal injection of pentobarbital (30 mg/kg, Virbac, Carros, France). The pancreas was dissected and the rats were euthanized by cervical dislocation. Then, the pancreas was immersed in $4 \%$ paraformaldehyde in phosphate-buffered saline (PBS), followed by overnight incubation in $30 \%$ sucrose solution. Paraffin-embedded pancreas tissue was serially sectioned at a thickness of $4 \mu \mathrm{m}$ on a microtome (Leica Microsystems, Wetzlar, Germany), followed by hematoxylin and eosin $(\mathrm{H} \& \mathrm{E})$ staining.

\section{Immunofluorescence microscopy}

To investigate whether EPA alters the immunosuppressive effect of CsA, rats were intraperitoneally injected with saline with or without $1.0 \mathrm{mg} / \mathrm{kg}$ lipopolysaccharide (LPS). Rats were euthanized $2 \mathrm{~h}$ later and the pancreas was dissected and fixed in $4 \%$ paraformaldehyde for $24 \mathrm{~h}$, then immersed in $30 \%$ sucrose for $48 \mathrm{~h}$ at $4^{\circ} \mathrm{C}$. After embedding in OCT compound (Sakura Finetek, Tokyo, Japan), samples were frozen in liquid nitrogen and stored at $-80^{\circ} \mathrm{C}$. The paraffinembedded tissue was serially cut into $30-\mu$ m-thick sections on a cryostat (Leica Microsystems).

The sections were permeabilized with $0.5 \%$ Triton $^{\mathrm{TM}} \mathrm{X}$ 100 for $10 \mathrm{~min}$ and then blocked with $10 \%$ goat serum (SigmaAldrich, St. Louis, MO, USA) in PBS, followed by antigen retrieval using Histo VT One (Nacalai Tesque, Kyoto, Japan). NFATc3 and nuclei were labeled with anti-NFATc3 antibody and TO-PRO-3, respectively. Five randomly selected sections per rat were imaged by confocal microscopy. Vacuolated pancreatic tissues were defined for each section. Samples were visualized with an LSM510 confocal microscope (Zeiss, Oberkochen, Germany).

\section{Statistical analysis}

Statistical analyses were performed using GraphPad Prism v.5.01 software (GraphPad Software Inc., La Jolla, CA, USA). Data were analyzed via one-way analysis of variance and multiple comparisons between treatment groups were made with the Dunnet's mulltiple comparison test or Bonferroni post hoc test. Results are presented as mean \pm SEM. A $\mathrm{P}$ value $\leq 0.05$ was considered statistically significant.

\section{RESULTS AND DISCUSSION \\ EPA reverses the decrease in glucose tolerance induced by CsA}

The effects of CsA and CsA + EPA administration for 2 weeks were evaluated by IPGTT. Consistent with a previous report (Lim et al., 2013), blood glucose level increased in the CsA group compared with that of untreated controls at each time point $(n=5$, Dunnett's multiple range test) (Fig. 1A). However, this increase was abrogated by co-administration of EPA.

These trends were confirmed by AUCg as an indicator of decreased glucose tolerance $(n=5$, Dunnet's multiple comparison test) (Fig. 1B).

\section{EPA abrogates pancreatic islet vacuolization caused by $\mathrm{CsA}$}

We examined whether long-term administration of CsA could damage pancreatic islet tissue by $\mathrm{H} \& \mathrm{E}$ staining (Fig. 2). CsA treatment for 2 weeks induced the formation of vacuoles in pancreas islets relative to untreated control rats. However, the number of vacuoles per islet was significantly reduced by coadministration of EPA compared with that observed with CsA alone, recovering almost to the level of the control group (control vs. CsA; P < 0.001, control vs. CsA + EPA; not significant. $\mathrm{n}=3$, Dunnett's Multiple range test). 

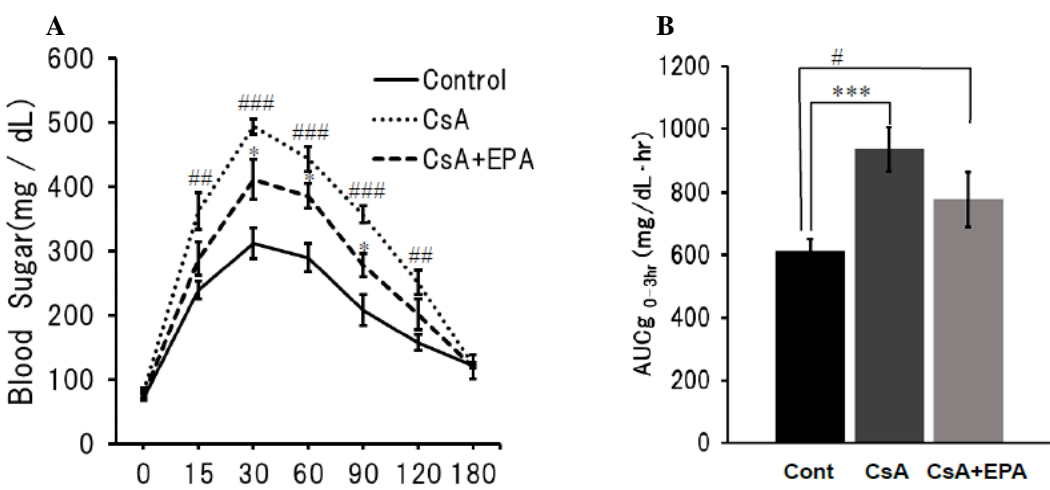

Fig. 1: Shift in blood glucose level and AUCg in the IPGTT. (A) rats were intraperitoneally injected with glucose and blood glucose level was monitored ${ }^{\#} \mathrm{P}<$ $0.05,{ }^{\#} \mathrm{P}<0.01,{ }^{\# \#} \mathrm{P}<0.001$, control vs CsA $* \mathrm{P}<0.05$, control vs. CsA + EPA ( $=5$ per group). (B) AUCg was determined with the trapezoidal method from pre-treatment to $3 \mathrm{~h}$ after glucose administration. $* * * \mathrm{P}<0.001$, control vs. CsA; ${ }^{\sharp} \mathrm{P}<0.05$, control vs. CsA + EPA ( $\mathrm{n}=5$ per group). Error bars indicate mean \pm SEM of independent experiments. Abbreviations: AUCg. Area under the curve glucose; IPGTT, intraperitoneal glucose tolerance test; CsA, cyclosporine A; EPA, eicospentaenoic acid.

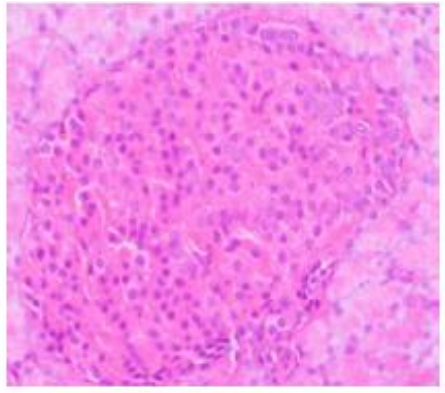

Control

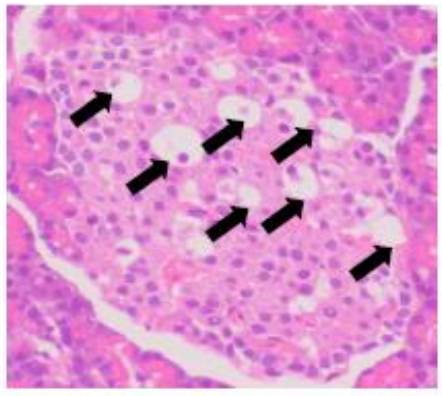

CsA

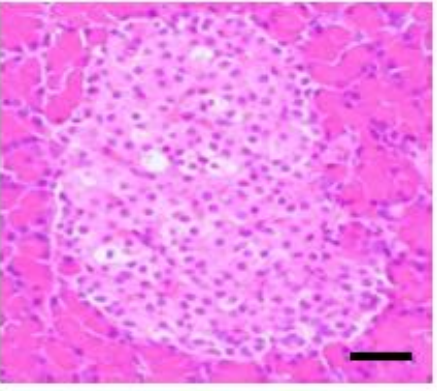

CsA+EPA

Fig. 2: Histological analysis of pancreatic islets. Pancreas tissue sections were stained with hematoxylin and eosin black arrows indicate vacuoles in pancreatic beta cells. Scale bars $50 \mu \mathrm{m}$. images are representative of three independent experiments.

\section{EPA does not alter the immunosuppressive effects of CsA}

NFATc3 is a transcription factor that regulates the expression of cytokines such as LPS-induced IL-2, and thereby plays an important role in the immune response. Treatment with CsA inhibited LPS-induced nuclear translocation of NFATc3 in the absence or presence of EPA ( $n=3$, Bonferroni post-hoc test) (Fig. 3B). These results suggest that EPA does not alter the ability of CsA to inhibit NFATc3 nuclear translocation, and, hence, does not impede immunosuppression by CsA. Although several studies have shown that omega-3 PUFAs have antioxidant and antiinflammatory effects (Gómez et al., 2004; Weylandt et al., 2008), the present work demonstrates for the first time that EPA treatment reverses damage to pancreatic islet cells caused by long-term administration of CsA in rats. Blood glucose levels increased 30 min after injection of glucose and returned to the baseline value after $180 \mathrm{~min}$ in all rats. However, CsA administration resulted in a persistent increase in blood glucose level at 15, 30, and $60 \mathrm{~min}$ relative to controls, which was suppressed by co-administration of EPA. This was confirmed by calculating AUCg $180 \mathrm{~min}$ after glucose administration and is consistent with previous reports that immunosuppressant-induced pancreatic dysfunction leads to hyperglycemia (Hahn et al., 1986, 1992; Piao et al., 2012). We also demonstrated that long-term administration of CsA induced vacuole formation, which was abrogated by EPA. This is in accordance with a report that oxidative stress or free radicals arising from chronic administration of immunosuppressants, such as CsA, caused pancreatic tissue dysfunction in a transplant patient (Lim et al., 2013). Based on these findings, we propose that elevation of blood glucose level upon long-term CsA administration is the result of pancreatic islet perforation, degeneration of pancreatic tissue, and consequent impairment in insulin secretion, and that EPA protects pancreatic tissue against these effects. Our immunofluorescence analysis revealed that nuclear translocation of NFATc3 in pancreatic cells induced by LPS was blocked by CsA in the absence or presence of EPA, indicating that EPA does not affect the immunosuppressive activity of CsA and that CsA-induced immunosuppression and pancreatic tissue damage are mediated via distinct mechanisms. CsA causes oxidative stress in the pancreas, leading to inflammation of islets. It was previously demonstrated that the antioxidant vitamins $\mathrm{C}$ and $\mathrm{E}$ could improve pancreatic disorder (Gómez et al., 2004). In addition to its antioxidant properties, EPA also suppresses inflammation and subsequent fibrosis (Weylandt $e t$ al., 2008; Oh et al., 2010). It was previously reported that $\mathrm{n}-3$ PUFAs, such as docosahexaenoic acid, more efficiently inhibited protein degradation than EPA by regulating inhibitor of $\kappa B \alpha$

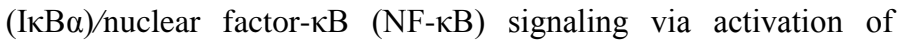
peroxisome proliferator-activated receptor- $\gamma$ gene expression (Wang et al., 2013); moreover, NF- $\mathrm{KB}$ activation in pancreatic acinar cells has been shown to exacerbate pancreatitis (Huang et al., 2013). Thus, EPA may suppress CsA-induced NF-kB activation by modulating I $\mathrm{KB} \alpha \mathrm{NF}-\kappa \mathrm{B}$ signaling. 


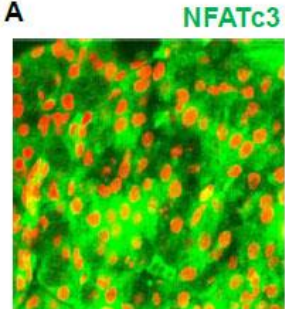

Vehicle

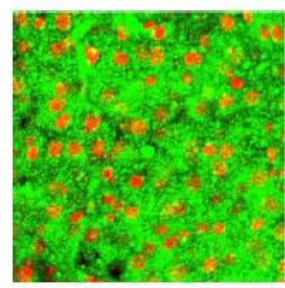

LPS + CSA

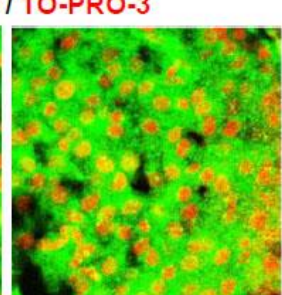

LPS

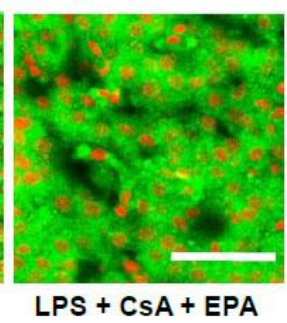

B

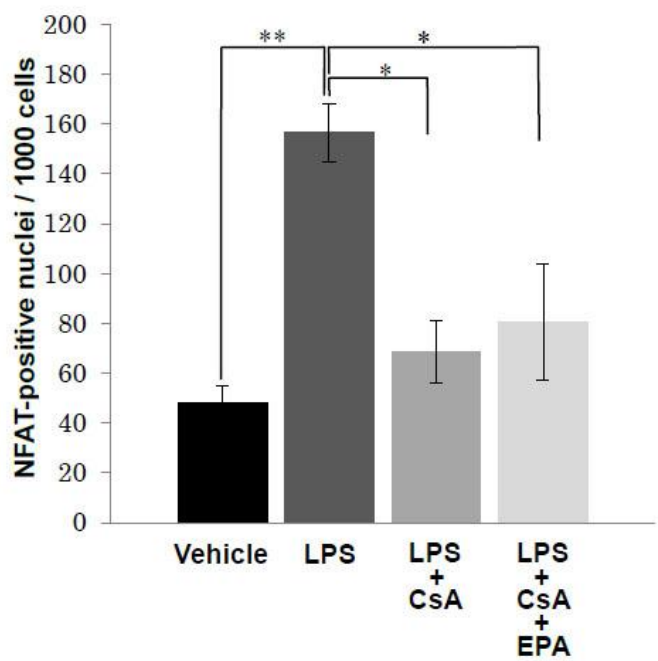

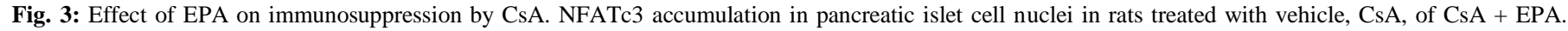

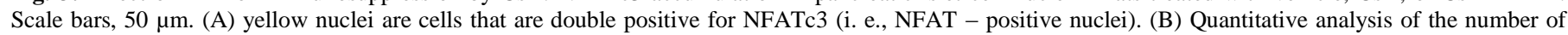

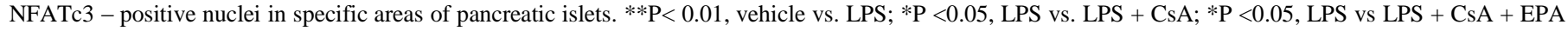

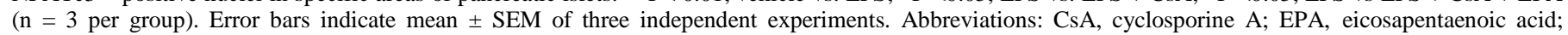
NFATc3, nuclear factor of activated T-cells 3.

\section{CONCLUSION}

In conclusion, our results indicate that EPA administration can prevent the adverse effects associated with CsA without affecting its immunosuppressive function in transplant patients.

\section{ACKNOWLEDGEMENT}

The authors thank K. Seki for providing expertise on immunohistochemical techniques and for helpful discussions, and the referees for useful comments.

\section{ETHICAL APPROVALS}

\section{Animal Rights}

The animal protocols used in this study were in accordance with the animal care guidelines of each institution and were approved by the local ethical committee (The Ohu University Medical School Animal Care and Use Committee, 2012-59, and 2013-29).

\section{Financial support and sponsorship: Nil.}

Conflict of Interests: There are no conflicts of interest.

\section{REFERENCES}

Chung BH, Li C, Sun BK, Lim SW, Ahn KO, Yang JH, Choi YH, Yoon KH, Sugawara A, Ito S, Kim J, Yang CW. Rosiglitazone protects against cyclosporine-induced pancreatic and renal injury in rats. Am J Transplant, 2005; 5:1856-1867.

Drachenberg CB, Klassen DK, Weir MR, Wiland A, Fink JC, Bartlett ST, Cangro CB, Blahut S, Papadimitriou JC. Islet cell damage associated with tacrolimus and cyclosporine: morphological features in pancreas allograft biopsies and clinical correlation. Transplantation, 1999; 68:396-402.
Düfer M, Krippeit-Drews P, Lembert N, Idahl LA, Drews G. Diabetogenic effect of cyclosporin $\mathrm{A}$ is mediated by interference with mitochondrial function of pancreatic B-cells. Mol Pharmacol, 2001; 60:873-879.

Gómez JA, Molero X, Vaquero E, Alonso A, Salas A, Malagelada JR. Vitamin E attenuates biochemical and morphological features associated with development of chronic pancreatitis. Am J Physiol Gastrointest Liver Physiol, 2004; 287:G162-G169.

Hahn HJ, Laube R, Lucke S, Besch W. Alteration of pancreatic B-cells in Wistar rats treated with non-diabetogenic doses of cyclosporin A. Pharmacol Toxicol, 1992; 70:188-191.

Hahn HJ, Laube F, Lucke S, Klöting I, Kohnert KD, Warzock R. Toxic effects of cyclosporine on the endocrine pancreas of Wistar rats. Transplantation, 1986; 41:44-77.

Heisel O, Heisel R, Balshaw R, Keown P. New onset diabetes mellitus in patients receiving calcineurin inhibitors: a systematic review and meta-analysis. Am J Transplant, 2004; 4:583-595.

Huang H, Liu Y, Daniluk J, Gaiser S, Chu J, Wang H, Li ZS, Logsdon CD, Ji B. Activation of nuclear factor- $\mathrm{kB}$ in acinar cells increases the severity of pancreatitis in mice. Gastroenterology, 2013; 144:202-210.

Jiang H, Sakuma S, Fujii Y, Akiyama Y, Ogawa T, Tamura K, Kobayashi M, Fujitsu T. Tacrolimus versus cyclosporin A: a comparative study on rat renal allograft survival. Transpl Int, 1999; 12:92-99.

Justo P, Lorz C, Sanz A, Egido J, Ortiz A. Intracellular mechanisms of cyclosporin A-induced tubular cell apoptosis. J Am Soc Nephrol, 2003; 14:3072-3080.

Lim SW, Doh KC, Jin L, Piao SG, Heo SB, Zheng YF, Bae SK, Chung $\mathrm{BH}$, Yang $\mathrm{CW}$. Oral administration of ginseng ameliorates cyclosporine-induced pancreatic injury in an experimental mouse model. PLoS One, 2013; 8:e72685.

Lim SW, Hyoung BJ, Piao SG, Doh KC, Chung BH, Yang CW. Chronic cyclosporine nephropathy is characterized by excessive autophagosome formation and decreased autophagic clearance. Transplantation. 2012 Aug 15;94(3):218-25.

Oh DY, Talukdar S, Bae EJ, Imamura T, Morinaga H, Fan W, Li P, Lu WJ, Watkins SM, Olefsky JM. GPR120 is an omega-3 fatty acid receptor mediating potent anti-inflammatory and insulin-sensitizing effects. Cell, 2010; 142:687-698.

Penfornis A, Kury-Paulin S. Immunosuppressive drug-induced diabetes. Diabetes Metab, 2006; 32:539-546. 
Piao SG, Bae SK, Lim SW, Song JH, Chung BH, Choi BS, Yang CW. Drug interaction between cyclosporine and mTOR inhibitors in experimental model of chronic cyclosporine nephrotoxicity and pancreatic islet dysfunction. Transplantation. 2012; 93(4):383-389.

Shimojo N, Jesmin S, Zaedi S, Maeda S, Soma M, Aonuma K, Yamaguchi I, Miyauchi T. Eicosapentaenoic acid prevents endothelin-1induced cardiomyocyte hypertrophy in vitro through the suppression of TGF- $\beta 1$ and phosphorylated JNK. Am J Physiol Heart Circ Physiol, 2006; 291:H835-H845.

Song HK, Han DH, Song JH, Ghee JY, Piao SG, Kim SH, Yoon HE, Li C, Kim J, Yang CW. Influence of sirolimus on cyclosporineinduced pancreas islet dysfunction in rats. Am J Transplant, 2009; 9:20242033.

Wang Y, Lin QW, Zheng PP, Zhang JS, Huang FR. DHA inhibits protein degradation more efficiently than EPA by regulating the PPAR $\gamma / \mathrm{NF} \kappa \mathrm{B}$ pathway in $\mathrm{C} 2 \mathrm{C} 12$ myotubes. Biomed Res Int, 2013; 2013:318981
Weylandt KH, Nadolny A, Kahlke L, Köhnke T, Schmöcker C, Wang J, Lauwers GY, Glickman JN, Kang JX. Reduction of inflammation and chronic tissue damage by omega- 3 fatty acids in fat- 1 transgenic mice with pancreatitis. Biochim Biophys Acta, 2008; 1782:634-641.

Yang CW, Faulkner GR, Wahba IM, Christianson TA, Bagby GC, Jin DC, Abboud HE, Andoh TF, Bennett WM. Expression of apoptosis-related genes in chronic cyclosporine nephrotoxicity in mice. Am J Transplant, 2002; 2:391-399.

\section{How to cite this article:}

Majima T, Sasaki K, Ohara H, Takahashi N. Eicosapentaenoic Acid Blocks Cyclosporine A-Induced Pancreatic Dysfunction But Not Immunosuppression. J App Pharm Sci, 2017; 7 (06): 001-005. 